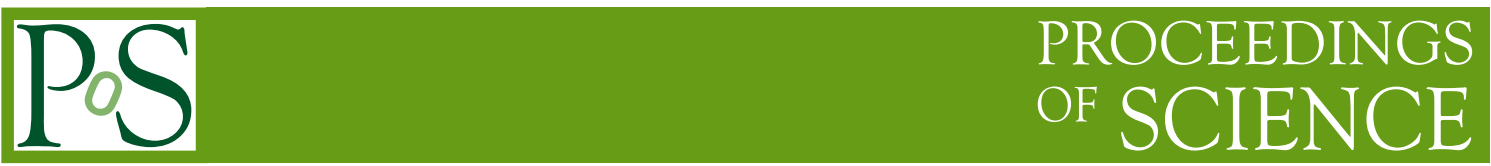

\title{
Searches for dark matter in hadronic final states with CMS
}

\author{
Deborah Pinna*† \\ Universität Zürich, Winterthurerstrasse 190, 8057 Zürich \\ E-mail: deborah.pinna@cern.ch
}

\begin{abstract}
Searches in CMS for dark matter in final states with invisible particles recoiling against hadronic final states are presented. Various topologies and kinematic variables are explored, as well as jet substructure as a means of tagging heavy bosons. The focus of the talk is the recent results obtained using data collected in 2016 run of the LHC.
\end{abstract}

The European Physical Society Conference on High Energy Physics

5-12 July, 2017

Venice

* Speaker.

${ }^{\dagger}$ On behalf of the CMS Collaboration. 


\section{Dark matter at colliders}

The standard model (SM) is a theory able to describe accurately almost all the known experimental observations in particle physics with high precision. Despite the accuracy of the SM, some phenomena are not naturally embedded in this theoretical framework. In particular, the SM does not provide any feasible dark matter (DM) candidate.

The evidence of the existence of DM is provided by astrophysical observations at different scales $[1,2,3]$. Nevertheless, information about its nature or non gravitational interactions is not yet available. Assuming interactions between DM and SM particles, DM can be produced in high energy collisions at the Large Hadron Collider (LHC) and its interactions studied in detail. Events with DM particles are rather challenging because the DM does not interact with the detector components. At hadron colliders, the presence of DM particle is therefore inferred as missing momentum balanced by a visible particle that is produced in association with the DM.

This contribution describes the most recent results from the CMS collaboration on DM in events with invisible particles recoiling against hadronic final states. These searches are performed using proton-proton collision data collected by the CMS experiment [4] in 2016 at a center of mass energy of $\sqrt{s}=13 \mathrm{TeV}$ and corresponding to an integrated luminosity of $35.9 \mathrm{fb}^{-1}$. An accurate interpretation of the results of these searches requires the inclusion of on-shell mediating particles into the particle spectrum in addition to DM. This approach, so called simplified model [5], assure an accurate description of the kinematic of the new phenomena including a minimal number of parameters: the DM particle mass, the mediator mass and the couplings $g_{\chi}$ and $g_{q}$ between the mediator and the DM particles or the SM quarks respectively.

\section{Search for dark matter in final states with an energetic jet or a hadronically decaying $\mathrm{W}$ or $\mathrm{Z}$ boson [6]}

In this search, the events are selected requiring for a large missing momentum and one or more high- $p_{T}$ jets. Based on the nature of such jets, events are then categorized in mono- $\mathrm{V}$ or mono-jet final states. An event falls in the former category if the selected jets are consistent with arising from the hadronic decay of Lorentz-boosted $\mathrm{W}$ or $\mathrm{Z}$ bosons. The compatibility with such hypothesis is verified considering the jet invariant mass computed with grooming techniques and the structure inside the jet. Events not qualifying for this category enter into the mono-jet final state. In both channels, the background is dominated by $\mathrm{Z} \rightarrow v v+$ jets and $\mathrm{W} \rightarrow 1 v+$ jets processes, which are estimated from data in dedicated control regions.

No significant excess over the SM prediction is observed, as shown for the mono-jet state on the left side of Fig. 1. Upper limits on the ratio of the excluded to predicted cross sections are set considering simplified models where the mediator is a vector, axial-vector, scalar, or a pseudoscalar particle. The center and left side of Fig. 1 show the results for the vector and axial-vector interaction hypotheses. In this search, the results are further interpreted in terms of invisible branching ratio of the Higgs boson or as limits on the parameters of fermion portal and light non-thermal DM models.

\section{Search for dark matter in final states with a top quark [7]}

The signature expected in this search consists in large missing momentum and a top quark 

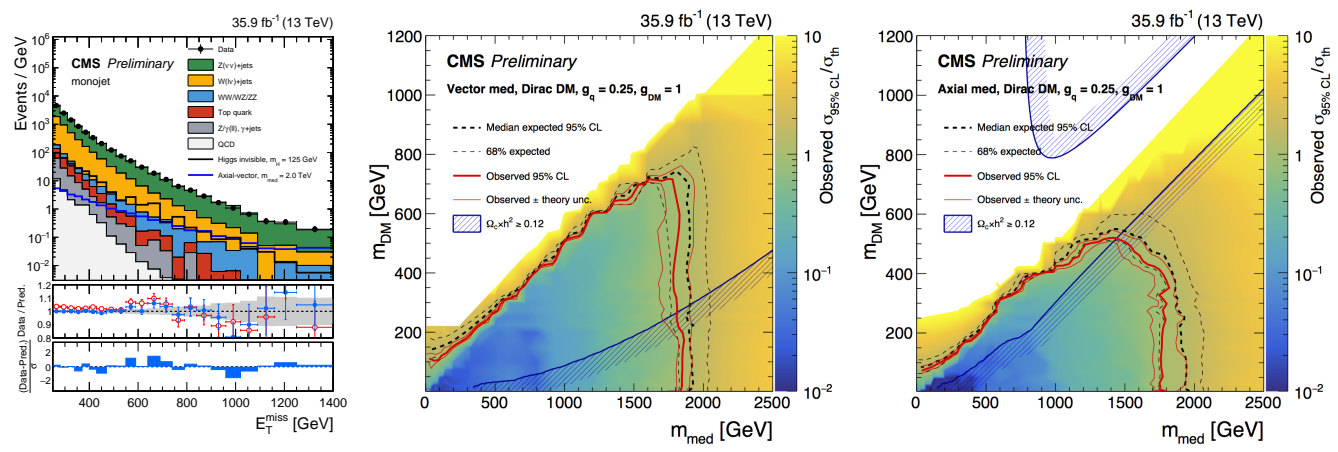

Figure 1: Missing transverse momentum $\left(E_{T}^{\text {miss }}\right)$ distribution in the mono-jet category (left) [6]. Upper limits on the ratio of the excluded to the predicted cross sections as a function of the DM and mediator masses for the vector (center) and axial-vector hypotheses (right) [6].

decaying hadronically. Events are selected requiring no leptons in the final state and the presence of a high- $p_{T}$ jet consistent with arising from a Lorentz-booosted top quark. In order to verify this condition the invariant mass of the jet is computed employing grooming techniques and the requirement of a b-tagged jet within the boosted jet is applied. In addition, three classes of substructure observables are employed as inputs into a boosted decision tree (BDT) to select events consistent with a 3-prongs structure within the boosted jet, as expected from the hadronic top quark decay. The BDT discriminant is used to divide the signal region into a loose and a tight category. In this search, the dominant background consists of $\mathrm{tt}, \mathrm{Z} \rightarrow v v+$ jets and $\mathrm{W} \rightarrow 1 v+$ jets events. These processes are estimated through data-driven methods employing dedicated control regions.

Data are found to be in good agreement with the SM prediction, as shown on the left side of Fig. 2 for the tight category. In the center and right side of Fig. 2, the results of this search are presented in terms of limits on the production cross section assuming two simplified models: production of DM in association with a single top quark via a neutral flavor-changing interaction (non-resonant model) or via the decay of a colored scalar resonance (resonant model). For the non-resonant model the results are also interpreted as a function of the mediator couplings with DM particles and quarks.
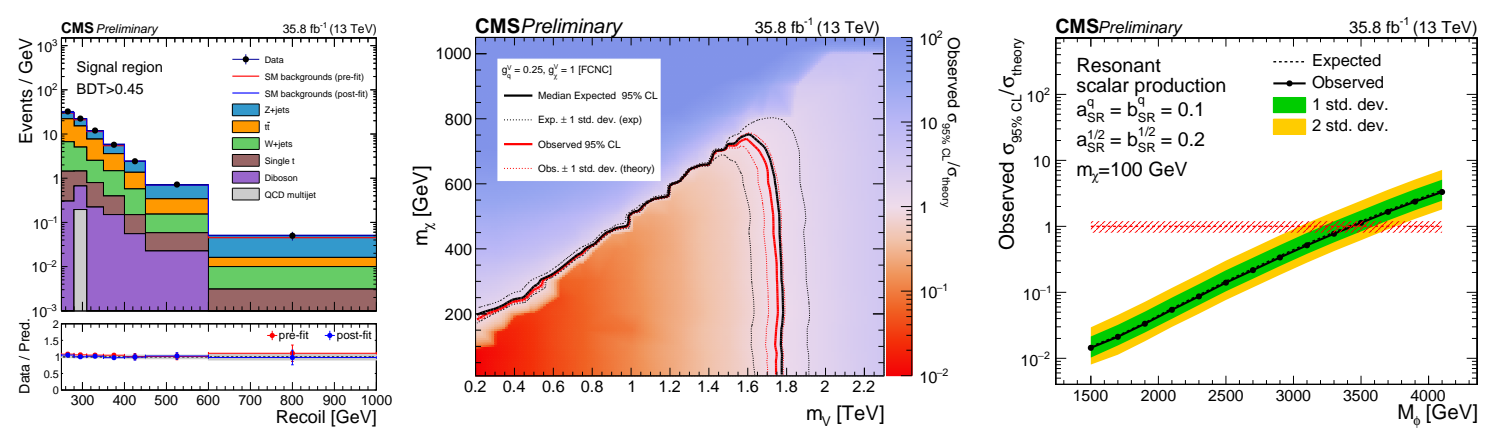

Figure 2: Missing transverse momentum distribution in the tight BDT category (left) [7]. Upper limits on the ratio of the excluded to the predicted cross sections as a function of the DM and mediator masses for the non-resonant (center) and resonant models (right) [7]. 


\section{DM mediator via dijet search}

In the simplified models employed to interpret DM searches at colliders the mediator of the interaction is considered to couple both to SM quarks and DM particles. Under this assumption, decays of the mediator into a pair of quarks are also possible. Depending on the value of the coupling to quarks, such mediator can be observed as a narrow or wide resonance, or through a non-resonant dijet signature.

Dijet searches are employed to investigate the production of narrow resonances, which will appear as a resonance in the dijet mass $\left(m_{j j}\right)$ spectrum above the smoothly falling background from QCD production of jets. The sensitivity to the mass of the dijet resonance below about $1 \mathrm{TeV}$ is limited by the overwhelming background and by trigger and storage limitations. The presented searches extend the reach in resonance masses below this limitation requiring an additional initial state radiation jet in the event [8] or employing events that are reconstructed, selected, and recorded in a compact format by the high-level trigger [9]. The dominant QCD multijet background is estimated in both searches with data-driven techniques. The $m_{j j}$ distributions in the signal regions are found to be in good agreement with the SM predictions. The results are then interpreted in terms of DM simplified models assuming a vector or axial-vector mediator following the recommendation of the LHC DM working group [10]. Fig. 3 shows the excluded values of the mediator mass as a function of the assumed DM mass for the two searches.

Wide resonances and non-resonant dijet signature are investigated employing the dijet angular distribution $\chi_{\text {dijet }}$ with respect to the beam direction [11]. Mediators not predicted by the SM are expected to appear as an excess of events at small values of $\chi_{\text {dijet }}$. The dijet angular distribution is measured and unfolded for detector effects in different dijet invariant mass regions. In each of these regions no significant excess over the theory predictions is observed. Results are interpreted in terms of DM simplified models and the excluded values for vector and axial-vector mediators are presented in Fig. 4 as a function of the DM mass.
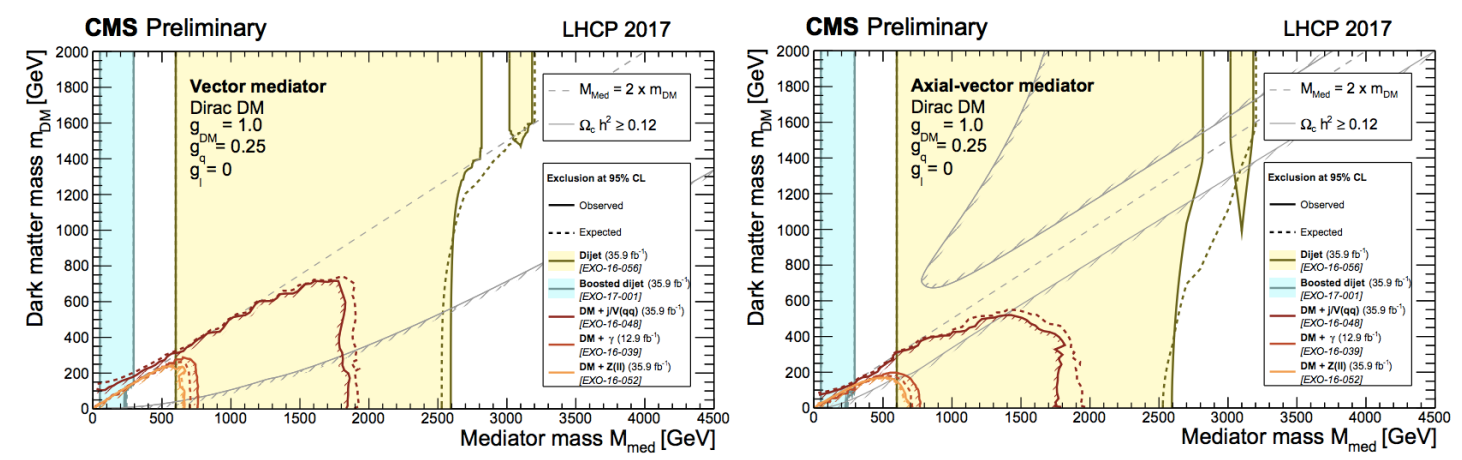

Figure 3: Observed and expected exclusion regions in the mediator and DM mass plane $\left(M_{m e d}-m_{D M}\right)$ for the presented dijet searches for the vector (left) and axial-vector (right) hypotheses [12].

\section{Summary}

The most recent results for dark matter in final states with invisible particles recoiling against hadronic final states performed by the CMS Collaboration have been presented. No significant 

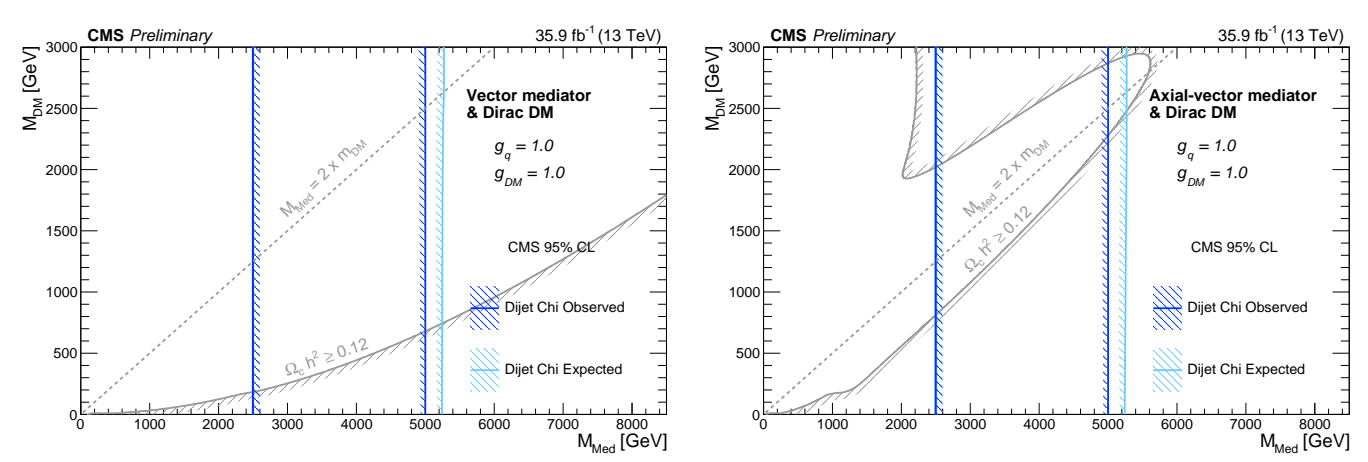

Figure 4: Observed and expected exclusion regions in the mediator and DM mass plane $\left(M_{\text {med }}-m_{D M}\right)$ for the presented dijet angular distribution search for the vector (left) and axial-vector (right) hypotheses [11].

excess over the SM background prediction is observed in the reported analyses. Limits on various simplified models have been set also exploiting the results from dijet searches.

\section{References}

[1] F. Zwicky, Die Rotverschiebung von extragalaktischen Nebeln, Helvetica Physica Acta 6, 110-127.

[2] V. C. Rubin and W. K. Ford, Jr., Rotation of the Andromeda Nebula from a Spectroscopic Survey of Emission Regions, Astrophys. J. 159 (1970), 379.

[3] D. Clowe, et al., A direct empirical proof of the existence of dark matter, Astrophys. J. 648 (2006), 109-L113.

[4] CMS Collaboration, The CMS Experiment at the CERN LHC, INST 3 S08004 (2008).

[5] Abercrombie, D. et al., Dark Matter Benchmark Models for Early LHC Run-2 Searches: Report of the ATLAS/CMS Dark Matter Forum, arXiv:1507.00966 [hep-ex].

[6] CMS Collaboration, Search for new physics in final states with an energetic jet or a hadronically decaying $W$ or $Z$ boson using $35.9 \mathrm{fb}^{-1}$ of data at $\sqrt{s}=13 \mathrm{TeV}$, Technical Report CMS-PAS-EXO-16-048 (2017), https : / / cds . cern. ch/record/ 2264698.

[7] CMS Collaboration, Search for dark matter in final states with a top quark and missing transverse momentum using new hadronic top quark tagging techniques, Technical Report

CMS-PAS-EXO-16-051 (2017), http: / / cds . cern. ch/record/2273454.

[8] CMS Collaboration, Search for light vector resonances decaying to a quark pair produced in association with a jet in proton-proton collisions at $\sqrt{s}=13 \mathrm{TeV}$, Technical Report CMS-PAS-EXO-17-001 (2017), https : / / cds. cern. ch/record/2264843.

[9] CMS Collaboration, Searches for dijet resonances in pp collisions at $\sqrt{s}=13 \mathrm{TeV}$ using data collected in 2016., Technical Report CMS-PAS-EXO-16-056 (2017),

https://cds.cern. ch/record/2256873.

[10] Albert, A. et al., Recommendations of the LHC Dark Matter Working Group: Comparing LHC searches for heavy mediators of dark matter production in visible and invisible decay channels, arXiv:1703.05703 [hep-ex].

[11] CMS Collaboration, Search for new physics with dijet angular distributions in proton-proton collisions at $\sqrt{s}=13 \mathrm{TeV}$ and constraints on dark matter and other models, Technical Report CMS-PAS-EXO-16-046 (2017), http: / / cds . cern. ch/record/2273455. 
[12] CMS Collaboration, Dark Matter Summary Plots from CMS for LHCP and EPS 2017, available at https://twiki.cern.ch/twiki/pub/CMSPublic/PhysicsResultsEXO/ DM-summary-plots-Jul17.pdf. 